Geistige Eigentumsrechte, was steht für die Entwicklungsländer auf dem spiel?

\title{
Geistige Eigentumsrechte : potentielle Auswirkungen auf Entwicklungsländer
}

\section{Carliene Brenner}

\section{(2) OpenEdition}

\section{Journals}

Electronic version

URL: http://journals.openedition.org/sjep/677

DOI: $10.4000 /$ sjep.677

ISSN: 1663-9677

\section{Publisher}

Institut de hautes études internationales et du développement

\section{Printed version}

Date of publication: 1 avril 1998

Number of pages: $7-23$

ISSN: $1660-5926$

\section{Electronic reference}

Carliene Brenner, « Geistige Eigentumsrechte : potentielle Auswirkungen auf Entwicklungsländer », Schweizerisches Jahrbuch für Entwicklungspolitik [Online], 17 | 1998, Online erschienen am: 01 August 2012, abgerufen am 08 September 2020. URL : http://journals.openedition.org/sjep/677 ; DOI : https://doi.org/10.4000/sjep.677 


\title{
GEISTIGE EIGENTUMSRECHTE: POTENTIELLE AUSWIRKUNGEN AUF ENTWICKLUNGSLÄNDER
}

\author{
CARLIENE BRENNER*
}

D

IE VORLIEGENDE Studie befasst sich mit potentiellen Auswirkungen der Stärkung der geistigen Eigentumsrechte aufgrund der Bestimmungen des Abkommens über handelsbezogene Aspekte der Rechte an geistigem Eigentum (TRIPS) auf die Entwicklungsländer. Der erste Abschnitt vermittelt einen Überblick über den Ausbau und die Ausweitung des Schutzes von geistigem Eigentum in den letzten Jahren. Darauf folgt eine Auseinandersetzung mit Art und Umfang der geistigen Eigentumsrechte sowie mit der Rolle der wichtigsten relevanten Organisationen. Der vierte Abschnitt nennt die hauptsächlichen Bestimmungen und Ausnahmen des TRIPS. Im fünften Abschnitt werden am Beispiel der Biotechnologie in der Landwirtschaft mögliche Auswirkungen einer Ausdehnung der geistigen Eigentumsrechte auf zuvor ungeschützte Bereiche - Mikroorganismen, Pflanzen- und Tiersorten - geprüft. Der letzte Abschnitt verweist auf die Schwierigkeit, eindeutige Schlussfolgerungen zu ziehen, und argumentiert, dass die Gesamtauswirkungen nicht ausschliesslich positiv oder negativ ausfallen werden. Zudem wird die Bedeutung einer staatlichen Politik zur Milderung etwaiger negativer Konsequenzen hervorgehoben.

\section{GEISTIGE EIGENTUMSRECHTE: VOM ALTERTUM BIS ZUM TRIPS}

Geistige Eigentumsrechte lassen sich definieren als Katalog von Gesetzen mit dem spezifischen Zweck, Erfinder und Schöpfer neuen Wissens zu schützen oder zu entschädigen. Da Wissen im Gegensatz zu materiellen Gütern ohne Substanzverluste von einer beliebigen Anzahl Personen geteilt werden kann, sind die Urheber auf rechtlichen Schutz angewiesen, um eine entschädigungslose direkte Nachahmung oder Verwendung zu verhindern. Der Zweck der geistigen Eigentumsrechte liegt darin, den Erfindern während eines beschränkten Zeitraums ausschliessliche Rechte zu verleihen (Lesser, 1994).

Das Konzept des Schutzes geistigen Eigentums lässt sich bis ins Altertum zurückverfolgen. Im alten Griechenland gewährte man Händlern und Erfindern bereits um 200 v. Chr. Monopolrechte. Auch im mittelalterlichen Europa (11. und 12. Jahrhundert), als das Handwerk und das Zunftsystem den Mittelpunkt von Herstellung und Vertrieb bildeten, wurden patentähnliche Vorrechte vergeben (Juma, 1989). Seit der industriellen Revolution haben verschiedene Formen des Patentschutzes eine weite Verbreitung erfahren. In Deutschland zum Beispiel wurde 1877 ein modernes Patentrecht verabschiedet. 
Patentgesetze sind in erster Linie nationale Gesetze, die sich je nach Land wesentlich unterscheiden. Erste Schritte zu einer internationalen Harmonisierung der geistigen Eigentumsrechte wurden mit der Pariser Verbandsübereinkunft zum Schutze des gewerblichen Eigentums von 1883 getan. Die Pariser Verbandsübereinkunft bildet einen Meilenstein in der Internationalisierung der geistigen Eigentumsrechte, vor allem weil sie den Grundsatz der «Nichtdiskriminierung» verankerte. Darunter ist zu verstehen, dass eine Vertragspartei den Staatsangehörigen anderer Länder denselben Schutz gewährt wie den eigenen Staatsangehörigen. Seit der Pariser Übereinkunft sind eine Reihe zusätzlicher internationaler Vereinbarungen über geistige Eigentumsrechte in Kraft getreten. $\mathrm{Zu}$ den wichtigsten zählen der Budapester-Verband, das Europäische Patentübereinkommen und der Vertrag über die internationale Zusammenarbeit auf dem Gebiet des Patentwesens'.

Im Jahr 1988 gab es in rund 115 Ländern einen Schutz geistiger Eigentumsrechte für gewerbliche Erfindungen. Dabei ist zu betonen, dass gegen 50 Prozent dieser Länder biologische Erfindungen - Pflanzen- und Tiersorten - vom Schutz ausschlossen (Lesser, 1991).

Der Schutz geistiger Eigentumsrechte für genetische Ressourcen von Pflanzen und für Pflanzensorten entwickelte sich unterschiedlich. Das hängt teilweise mit der Komplexität und Schwierigkeit des Schutzes der lebendigen Materie zusammen. Ein wichtiger Fortschritt zur Entwicklung der Schutzrechte wurde in den 30er Jahren dieses Jahrhunderts in den Vereinigten Staaten mit der Einführung der HybridSorten von Mais verwirklicht. Die neuen Hybrid-Züchtungen lieferten zwar höhere Erträge als offen bestäubte Sorten, aber die Aufbewahrung für eine zweite Aussaat war mit einem drastischen Ertragsrückgang verbunden. So mussten die Landwirte, die Saatgut im Betrieb gelagert hatten, für jede Saatzeit die neuen Sorten kaufen, um weiterhin höhere Erträge zu erzielen. Dies verschaffte den Erfindern oder Züchtern von Hybrid-Sorten einen patentähnlichen Schutz.

Mit der Entwicklung der Agrarforschung und der modernen Pflanzenzüchtung erhoben auch die Pflanzenzüchter Anspruch auf geistiges Eigentum und auf Schutz der Ergebnisse ihrer Arbeit - mit der Begründung, ihr gesellschaftlicher Beitrag müsse gleich anerkannt werden wie jener der gewerblichen Erfinder.

Im Anschluss an die Pariser Verbandsübereinkunft führten die Forderungen der Pflanzenzüchter nach Pflanzenschutz in einigen europäischen Ländern in den 20er Jahren, in den Vereinigten Staaten in den 30er Jahren zur Verabschiedung von Gesetzen über den Schutz neuer Pflanzenzüchtungen. Der amerikanische «Plant Patent Act» (Gesetz über Patentschutz für Pflanzen) von 1930 sah einen patentrechtlichen Schutz für ungeschlechtlich vermehrte Pflanzen vor (ausgenommen Knollen). Geschlechtlich vermehrte Pflanzen sind jedoch ausgeschlossen, da sie die Besonderheit aufweisen, sich im Laufe der Generationen zu entwickeln und zu verändern, was die Bestimmung des ursprünglichen Patentgegenstandes erschwert.

1. Der Vertrag von 1997 über die internationale Anerkennung der Hinterlegung von Mikroorganismen für die Zwecke von Patentverfahren bezieht sich auf die Anerkennung der Hinterlegung lebender Organismen in international anerkannten Einrichtungen. Das Europäische Patentübereinkommen (EPÜ) gilt für die Mitgliedsstaaten der Europäischen Union. Der PCT-Vertrag bezweckt mit der einheitlichen internationalen Anmeldung in einem Mitgliedsstaat, der darauffolgenden internationalen Veröffentlichung und dem internationalen Recherchenbericht eine Vereinfachung des Patentierens in den verschiedenen Ländern und eine Kostensenkung. Diese internationalen Patentabkommen werden von der Weltorganisation für geistiges Eigentum (WIPO) verwaltet. 
Erste internationale Bemühungen um eine Erweiterung und Harmonisierung des Züchterrechts- auch als Sortenschutzrecht bezeichnet - erfolgten auf dem Kongress des Internationalen Verbandes der Pflanzenzüchter zum Schutz von Pflanzensorten (Association Internationale des Sélectionneurs pour la Protection des Obtentions Végétales ASSINSEL) von 1956 in Österreich. Dies führte 1961 zum ersten Internationalen Übereinkommen zum Schutz von Pflanzenzüchtungen (unter der französischen Abkürzung UPOV für Union pour la Protection des Obtentions Végétales bekannt).

Die verschiedenen Arten geistiger Eigentumsrechte für gewerbliche und landwirtschaftliche Technologie entwickelten sich zwar getrennt weiter. Gemeinsam war allen Innovationsbereichen eine allmähliche, doch markante Stärkung des Schutzes des geistigen Eigentums im Laufe der Jahre. Teilweise erklärte sich diese Entwicklung aus der Unzufriedenheit der Patentinhaber über Verluste, die auf Verletzungen geistiger Eigentumsrechte zurückgingen. Die Internationale Trade Commission (Handelsbehörde) der Vereinigten Staaten schätzte die Einkommenseinbussen, die Erfindern in Industrieländern wegen Patentverletzungen entstehen, für amerikanische Unternehmen 1990 auf 61 Milliarden US\$ jährlich (Lesser, 1991).

Zusätzlich zur Stärkung des Schutzes des geistigen Eigentums zeichnete sich zumindest in den OECD-Ländern mit dem Durchbruch der Biotechnologie auch eine Konvergenz der Schutzarten der gewerblichen und biologischen Erfindungen ab. Ein wesentlicher Schritt in diese Richtung bildete die zukunftsweisende Entscheidung des amerikanischen Supreme Court (Oberstes Gericht) in Sachen Chakrabarty von 1980, welche erstmals das Patentieren von gentechnisch modifizierten Organismen - oder Lebensformen - erlaubte. Der erste Patentantrag für genmodifizierte Pflanzen wurde 1983 eingereicht. Das erste gewerbliche Patent für eine Pflanzensorte erteilten 1985 die Vereinigten Staaten, das erste Patent für eine gentechnisch veränderte Pflanze wurde 1988 in Europa gewährt. In den 80er Jahren stieg die Zahl der Patentanträge im Bereich der Pflanzen-Biotechnologie auf rund 250 pro Jahr an (Joly, de Looze, 1996). (Die Behandlung von Art und Umfang der geistigen Eigentumsrechte in Abschnitt 2 veranschaulicht, dass die Revision des UPOV-Übereinkommens von 1991 auch zu einer weiteren Annäherung von Sortenschutz und Patenten führte).

Weil die Vereinigten Staaten nachdrücklich betonten, das Fehlen eines umfassenden Patent- und geistigen Eigentumsrechts bilde ein nicht-tarifäres Handelshemmnis, wurde das «handelsbezogene geistige Eigentum» trotz des Widerstands der Entwicklungsländer in die unter der Ägide des Allgemeinen Zoll- und Handelsabkommens (GATT) lancierten multilateralen Handelsverhandlungen der Uruguay-Runde einbezogen. Die Bemühungen um eine Stärkung und Erweiterung der geistigen Eigentumsrechte mündeten in der Unterzeichnung des TRIPS-Abkommens (Anhang 1C des Abkommens von Marrakesch vom 1. Januar 1995 zur Errichtung der Welthandelsorganisation WTO). Der Schwerpunkt der Verhandlungen und der Diskussion zu geistigen Eigentumsrechten hat sich demnach von den technischen Arbeiten der Weltorganisation für geistiges Eigentum (WIPO), einem UN-Organ, zur neu geschaffenen WTO verlagert.

Die Konsequenzen, die eine Stärkung der geistigen Eigentumsrechte und eine Ausdehnung des Schutzes auf zuvor nicht abgedeckte Bereiche - Mikroorganismen, genetisches Material von Pflanzen, Genmanipulationstechniken - insbeson- 
dere für Entwicklungsländer haben könnten, beherrschen heute eine lebhafte Debatte. Die Problematik ist in bezug auf die Umweltzerstörung und den notwendigen Schutz der biologischen Vielfalt von grösster Bedeutung. In formaler Hinsicht sind diese Themen bei dem «International Undertaking on Plant Genetic Resources IUPGR» der FAO und beim Übereinkommen für biologische Vielfalt untergebracht. Im IUPGR der FAO wird der Schutz geistiger Eigentumsrechte und pflanzlichen Genmaterials mit den Rechten der Landwirte ( "farmers'rights ») verknüpft, während das Übereinkommen über die biologische Vielfalt diesen Schutz mit dem Ziel einer «ausgewogenen und gerechten Aufteilung der sich aus der Nutzung der genetischen Ressourcen ergebenden Vorteile» verbindet.

\section{HAUPTFORMEN UND UMFANG DER GEISTIGEN EIGENTUMSRECHTE}

Die wichtigsten Formen der geistigen Eigentumsrechte umfassen Patente, Züchterrechte und Geschäftsgeheimnisse. Urheberrechte und Marken gehören ebenfalls dazu, werden aber in dieser Studie nicht behandelt.

\subsection{PATENTE}

Patente, einschliesslich Gebrauchsmuster, sind die verbreitetste und gleichzeitig die älteste Form des Schutzes von geistigem Eigentum. Jede nicht ausdrücklich verbotene Erfindung ist patentfähig, vorausgesetzt, dass sie die unten beschriebenen Anforderungen erfüllt. Entdeckungen, wissenschaftliche Theorien und mathematische Formeln sowie Gegenstände, die gegen die guten Sitten verstossen, sind von der Patentfähigkeit ausgeschlossen. Immer mehr Länder gewähren Patente für eine breite Palette von Erfindungen - Erzeugnisse und Verfahren -, wozu auch ausgewähltes lebendes Material wie DNA-Sequenzen, Gene, Mikroorganismen, Pflanzenteile, Pflanzen- und Tiersorten gehören. Zahlreiche Entwicklungsländer und einige OECD-Mitgliedsstaaten haben Arzneimittel und Produkte, die sich auf die Landwirtschaft beziehen, von der Patentierbarkeit ausgeschlossen.

Die Erteilung eines Patents ist an drei wichtige Bedingungen geknüpft: Nützlichkeit oder gewerbliche Anwendung; Neuheit oder Neuartigkeit im Sinn, dass die Erfindung zuvor nicht allgemein bekannt war; und Nicht-Offensichtlichkeit oder erfinderische Tätigkeit (inventive step), d.h. die Erfindung muss eine anerkannte Bereicherung des Wissens bedeuten.

Nach dem Kriterium der Nützlichkeit können blosse Ideen oder Konzepte nicht patentiert werden. Eine Erfindung muss die Komponente der menschlichen Anstrengung beinhalten und im Fall einer Anwendung erwartungsgemäss funktionieren. Die Voraussetzung der Neuartigkeit oder Neuheit bedeutet, dass die Erfindung vor dem Patentantrag nicht öffentlich bekannt war und insofern eine Entdeckung für die Menschheit darstellt.

Als zusätzliches Erfordernis für die Patenterteilung gilt die Offenbarung: Die Erfindung muss in der Patentanmeldung so deutlich und vollständig beschrieben (offenbart) werden, dass ein Fachmann in der Lage ist, das Patent selbst herzustellen bzw. zu verwenden oder die Erfindung selbst nachzuvollziehen (Lesser, 1991). Für immer zahlreichere Erfindungen in der Mikrobiologie reicht die 
Offenbarung allein nicht mehr aus. Zusätzlich wird die Hinterlegung einer Probe des biologischen Materials, von dem die Erfindung abgeleitet wurde, verlangt.

Im allgemeinen haben die nationalen Patentämter sicherzustellen, dass die erforderlichen Voraussetzungen eingehalten werden, und sind für die Erteilung oder Verweigerung der Patente verantwortlich. Die Entscheidung, ein Patent zu erteilen bzw. zu verweigern, beruht entweder auf der Prüfung oder auf der Eintragung. Bei einer Prüfung führen die nationalen Patentämter eine Recherche durch, um sicherzustellen, dass die beanspruchte Erfindung tatsächlich neu ist. Der Anspruch wird auch geprüft, um zu ermitteln, ob die Erfindung in bezug auf den früheren Wissensstand hinreichend unterschiedlich (oder nicht offensichtlich) ist, um eine echte Erfindung darzustellen. Bei einer Eintragung wird die Anmeldung entgegengenommen und registriert, ohne die Gültigkeit des Patents zu beurteilen.

Die Patenterteilung verleiht dem Inhaber oder Erfinder während eines begrenzten Zeitraums ausschliessliche Rechte über die Verwendung und den Gewinn aus der Erfindung. Die Schutzdauer beträgt - mit landesspezifischen Abweichungen - im allgemeinen 14 bis 20 Jahre. (Für Gebrauchsmuster ist diese Dauer kürzer, sie beläuft sich im Regelfall auf 4 bis 7 Jahre). Während dieser Zeit hat der Erfinder das Recht, anderen Herstellung, Verwendung, Verkauf, Feilhalten oder Einfuhr der Erfindung zu verbieten oder für die Verwendung (Lizenz) eine Gebühr zu erheben (Lesser, 1991).

In bestimmten Fällen kann eine Lizenz zur Verwendung einer Erfindung ohne Genehmigung des Patentinhabers erteilt werden. «Zwangslizenzen» können bei «Unzulänglichkeit» des Patents erteilt werden oder wenn ein öffentliches Interesse angenommen wird (insbesondere im Zusammenhang mit Gesundheit, Ernährung und Sicherheit) (Lesser, 1991).

\subsection{SORTENSCHUTZRECHT}

Während die meisten Länder - mit der wichtigen Ausnahme der Vereinigten Staaten - Erfindungen mit Pflanzen vom Gebrauchspatentschutz ausklammern, sehen immer mehr Staaten mit dem Sortenschutz eine andere Schutzart für geistiges Eigentum vor. In Übereinstimmung mit dem UPOV-Übereinkommen, das 1961 in Kraft trat und 1972, 1978 und 1991 revidiert wurde, beschränkt sich der Schutzumfang des Sortenschutzes auf neue Sorten. ${ }^{2}$

Diese Rechte des Züchters verhindern eine nicht bewilligte Verwendung der geschützten Sorten. Die Schutzvoraussetzungen für Pflanzensorten sind ähnlich wie für Gebrauchspatente, allerdings weniger umfassend: Neuheit, Unterscheidbarkeit, Einheitlichkeit oder Homogenität und Beständigkeit. Ferner muss eine Sorte zur Identifikation eine Bezeichnung erhalten.

2. «Sorte» bedeutet « eine pflanzliche Gesamtheit innerhalb eines einzigen botanischen Taxons der untersten bekannten Rangstufe, die unabhängig davon, ob sie voll den Voraussetzungen für die Erteilung eines Züchterrechts entspricht,

- durch die sich aus einem bestimmten Genotyp oder einer bestimmten Kombination von Genotypen ergebende Ausprägung der Merkmale definiert werden kann,

- zumindest durch die Ausprägung eines der erwähnten Merkmale von jeder anderen pflanzlichen Gesamtheit unterschieden werden kann und

- in Anbetracht ihrer Eignung, unverändert vermehrt zu werden, als Einheit angesehen werden kann.»

Artikel 1 der Akte des UPOV-Übereinkommens von 1991. 
Neuheit wird dadurch bestimmt, dass eine Sorte im Land, in dem der Antrag eingereicht wird, oder in einem anderen Land nicht länger als vier Jahre verkauft oder anders abgegeben wurde. Die Voraussetzung der Unterscheidbarkeit - die wichtigste Basis für die Erteilung des Züchterrechts - gilt als erfüllt, wenn die Sorte sich von jeder anderen Sorte, deren Vorhandensein allgemein bekannt ist, in einem oder mehreren wichtigen Merkmalen deutlich unterscheiden lässt. Homogenität beinhaltet, dass eine Sorte in ihren massgebenden Merkmalen einheitlich ist. Beständigkeit schliesslich bedeutet, dass die neue Sorte nach aufeinanderfolgenden Vermehrungen unverändert bleibt. ${ }^{3}$

Das Züchterrecht gilt zwar als schwächere Schutzform für geistiges Eigentum als Patente. Doch der Schutzumfang für Pflanzenzüchter konnte mit jeder Revision des UPOV-Übereinkommens ausgebaut werden. Die jüngste Fassung von 1991 unterscheidet sich in folgenden Punkten deutlich von der früheren Version von 1978: Schutzinhalt und Schutzdauer, Rechte des Pflanzenzüchters, «farmers'privilege» (Landwirteprivileg), Konzept der «im wesentlichen abgeleiteten Sorte».

Gemäss der Akte von 1991 (Artikel 3) werden die Staaten aufgefordert, alle Pflanzengattungen und -arten zu schützen; die Akte von 1978 beschränkte sich auf eine begrenzte Anzahl Arten. Staaten, die bereits Verbandsmitglieder sind, gewähren diesen höheren Schutzumfang spätestens nach fünf Jahren. Für neue Verbandsmitglieder wird die Frist auf 10 Jahre erstreckt. Zur Schutzdauer sieht die Akte von 1991 eine Zeit von 25 Jahren für Bäume und Reben und von 20 Jahren für alle anderen Pflanzen vor, verglichen mit 18 bzw. 15 Jahren in der Akte von 1978.

Nach der Akte von 1978 umfasst das Züchterrecht die Erzeugung und den Verkauf von generativem oder vegetativem Vermehrungsmaterial einer neuen Sorte. In der Akte von 1991 wird dieses Recht auf das Erntegut (beispielsweise die Frucht einer geschützten Obstbaumsorte) ausgedehnt - in anderen Worten auf die gesamte Erzeugung und Vermehrung der geschützten Sorte. Allerdings können die Länder auf freiwilliger Basis für die traditionellen Formen der Aufbewahrung von Saatgut im Betrieb eine Ausnahme vom Züchterrecht vorsehen.

Das Züchterrecht gilt zwar für die Herstellung zu kommerziellen Zwecken, aber nicht für die Herstellung von Vermehrungsmaterial, das nicht für den kommerziellen Vertrieb bestimmt ist. So können die Landwirte Saatgut für eine spätere Aussaat im eigenen Betrieb gewinnen. Diese Praxis fällt nicht unter den Schutz des Züchterrechts und wird als «farmers'privilege» bezeichnet.

Sowohl die Akte von 1978 als auch jene von 1991 sehen die sogenannte «breeders'exemption» (Züchtervorbehalt) vor, wonach eine geschützte Sorte ohne Zustimmung des Züchters zu Versuchszwecken oder als Ursprungssorte zur Schaffung neuer Sorten verwendet werden darf. Allerdings führt die Akte von 1991 das Konzept der «wesentlichen Ableitung» ein: «im wesentlichen abgeleitete» Sorten einer geschützten Sorte können ohne Zustimmung des Züchters ihrer geschützten Ursprungssorte zwar geschützt, aber nicht kommerziell verwendet werden. ${ }^{4}$

3. Siehe Artikel 6, 7, 8 und 9 des UPOV-Übereinkommens.

4. Mit der gentechnischen Transformation wurde es theoretisch möglich, eine beliebige Sorte im Laboratorium durch das Hinzufügen von einem oder mehreren Genen zu verändern und die veränderte Sorte zu schützen, sofern sie sich deutlich von der Ursprungssorte unterscheiden liess - ohne Verpflichtung des Veränderers gegenüber dem ursprünglichen Züchter. Siehe Why change the UPOV Convention? The Evolution of the Convention resulting from the 1991 Act, Ausführungen von Adelaide Harries am «Information Meeting on the Protection of New Varieties of Plants under the UPOV Convention, Rome, April 19, $1996 »$. 
Gegenwärtig gilt noch die UPOV-Akte von 1978. Die Akte von 1991 wird einen Monat nach dem Zeitpunkt in Kraft treten, in dem fünf Staaten ihre Beitrittsurkunden hinterlegt haben, wobei mindestens drei Urkunden von Verbandsmitgliedern der früheren Akte stammen. Zuerst wurde vereinbart, nach dem Inkrafttreten der Akte von 1991 keine Beitritte zur Akte von 1978 mehr zu erlauben. Da jedoch mehrere Länder die Beitrittsverfahren auf der Grundlage der Akte von 1978 eingeleitet hatten, wurde beschlossen, dass diese noch ein Jahr nach dem Inkrafttreten der Akte von 1991 zum Beitritt offenstehen soll. Im Juni 1997 waren erst drei Länder der Akte von 1991 beigetreten: Dänemark, die Niederlande und Israel.

\subsection{GESCHÄFTSGEHEIMNISSE}

Geschäftsgeheimnisse lassen sich definieren als vertraulich behandelte, nicht öffentlich bekanntgemachte Informationen, die dem Inhaber einen Wettbewerbsvorteil verleihen. In den meisten Ländern fehlt eine rechtliche Definition des Geschäftsgeheimnisses. Allerdings sehen die Gesetze bei unlauterem Erwerb von gewerblichen Geheimnissen den Rechtsweg vor, um eine weitere Verwendung zu verhindern und/oder Schadenersatz zu verlangen. Geschäftsgeheimnisse werden in der Regel durch das Verbot des Informationszugangs in Verträgen oder Vereinbarungen geschützt. Dank den Geschäftsgeheimnissen können bestimmte nicht-kodifizierte Aspekte der Technologie - die «implizite» Technologie - weiter kontrolliert werden, was den Wert eines Patentes erhöht. So geschieht der Verkauf oder Transfer von komplexen Technologien oft in Form eines Pakets, welches eine oder mehrere Patentlizenzen, Geschäftsgeheimnisse und Managementberatung umfasst (Lesser, 1991).

Die Elterngenerationen von Hybriden oder die in gentechnischen Manipulationen verwendeten Reagenzien werden tendenziell eher durch Geschäftsgeheimnisse als durch Patente oder durch den Sortenschutz geschützt. Oft versuchen Unternehmen, ihre Technologie durch Geschäftsgeheimnisse zu schützen, wenn noch kein anderer Schutz besteht.

\subsection{MATERIALTRANSFERABKOMMEN (MTA)}

MTA werden vorwiegend zum Transfer von genetischem Material zu Forschungszwecken abgeschlossen. Eine wichtige Rolle spielen sie in der Teamforschung bei öffentlich finanzierten oder gesponserten Forschungsprojekten und programmen mit Universitäten und/oder öffentlichen Forschungseinrichtungen als Partnern, aber auch in der Forschung mit Partnern aus der Privatwirtschaft.

\section{AUFGABEN DER WICHTIGSTEN ORGANISATIONEN IM BEREICH DES GEISTIGEN EIGENTUMS}

Das TRIPS-Abkommen schafft keine Ausnahmen von bestehenden Verpflichtungen, welche die Unterzeichnerstaaten gegebenenfalls mit den Bestimmungen der Pariser Verbandsübereinkunft (zum Schutz des gewerblichen Eigentums), der Berner Übereinkunft (zum Schutz von Werken der Literatur und Kunst), des 
Rom-Abkommens (über den Schutz der ausübenden Künstler, der Hersteller von Tonträgern und der Sendeunternehmen) oder des Vertrags über den Schutz des geistigen Eigentums an integrierten Schaltkreisen übernommen haben. Die WTO hat sich heute zum Verhandlungsforum im Bereich der geistigen Eigentumsrechte entwickelt. Andere relevante Organisationen wie die Weltorganisation für geistiges Eigentum (WIPO) oder die UPOV erfüllen jedoch weiterhin eine wesentliche, ja sogar wachsende Rolle.

Die 1967 gegründete WIPO wurde 1974 zu einer Sonderorganisation im System der Vereinten Nationen. Die WIPO hat die Aufgabe, den Schutz des geistigen Eigentums weltweit durch eine Zusammenarbeit der Staaten und - soweit sinnvoll - im Zusammenwirken mit anderen internationalen Organisationen zu fördern. Ferner gewährleistet die WIPO die administrative Zusammenarbeit der Verbände für geistiges Eigentum, der «Unionen» also, die durch die Übereinkommen von Paris und von Bern sowie durch verschiedene Unterverträge der Mitglieder der Pariser Verbandsübereinkunft geschaffen wurden.

Die WIPO bietet Entwicklungsländern im Bereich des geistigen Eigentums eine breite Palette von Dienstleistungen an. Dazu gehören Beratung und Ausbildung für Regierungen und Organisationen des öffentlichen und privaten Sektors zu Verhandlungen und Vereinbarungen in Fragen der Lizenzerteilung für geistiges Eigentum und des «Managements » solcher Vereinbarungen, sofern diese ökologische Auswirkungen haben. Ferner erarbeitet die WIPO Berichte zum jüngsten Stand der Forschung in verschiedenen Technologiesektoren. Diese Forschungsberichte beruhen auf den Informationen, die in Patentunterlagen veröffentlicht werden, und sind gebührenfrei erhältlich.

Das am 1. Januar 1996 zwischen der WIPO und der WTO abgeschlossene Abkommen sieht vor, dass die WIPO weiterhin rechtliche und technische Unterstützung und technische Zusammenarbeit zugunsten der Entwicklungsländer leisten soll. Diese Unterstützung soll gestützt auf das TRIPS-Abkommen auch Entwicklungsländer erfassen, welche Mitglieder der WTO, aber nicht unbedingt der WIPO sind.

Die Arbeiten der WIPO im Interesse der Entwicklungsländer konzentrieren sich wie in der Vergangenheit auf das Gebiet des gewerblichen Rechtsschutzes. Der Schutz von Pflanzensorten bleibt so bei der UPOV. Da immer mehr Länder den Sortenschutz den Pflanzenpatenten vorziehen, wird die UPOV eine wichtigere Rolle übernehmen.

Während die WTO-Mitgliedschaft und damit die Annahme des TRIPS-Abkommens nahezu universal sind, zählte die UPOV im August 1997 nur 34 Mitgliedstaaten. Dazu gehörten die meisten OECD-Staaten, ausgenommen Griechenland, Island, Korea, Luxemburg und die Türkei. Als einzige Nichtmitglieder der OECD gehören derzeit Argentinien, Chile, Kolumbien, Ecuador, Israel, Paraguay, die Slowakei, Südafrika, die Ukraine und Uruguay zur UPOV.

Eine steigende Anzahl von Ländern in Osteuropa, Lateinamerika, im Nahen Osten, in Afrika und Asien sind heute dabei, den Beitritt zur UPOV vorzubereiten bzw. ihre Gesetze über den Schutz von Saatgut und Pflanzen zu revidieren. Einige Länder entscheiden sich für die Bestimmungen der Akte von 1978, andere für jene von 1991. Die UPOV-Mitgliedschaft dürfte sich so rasch ausweiten. Für 1999 werden rund 50 Länder, für 2000 rund 80 Länder erwartet. 


\section{TRIPS-BESTIMMUNGEN UND AUSNAHMEN}

Das TRIPS-Abkommen, ein Meilenstein in der Geschichte des geistigen Eigentums, ist am 1. Januar 1995 in Kraft getreten. Es handelt sich um die erste weltweite Bemühung, die Länder auf spezifische Normen hinsichtlich Verfügbarkeit, Tragweite und Verwendung geistiger Eigentumsrechte $\mathrm{zu}$ verpflichten. Das Abkommen deckt alle Hauptgebiete des geistigen Eigentums ab: Urheberrechte und verwandte Schutzrechte, Marken, geographische Angaben, gewerbliche Muster, Patente, Layout-Designs (Topographien) integrierter Schaltkreise und vertrauliche Informationen.

Die Ziele des Abkommens (Artikel 7 TRIPS) lauten wie folgt: Der Schutz und die Durchsetzung der Rechte an geistigem Eigentum sollen zur Förderung der technischen Innovation sowie zum Transfer und zur Verbreitung von Technologie beitragen; und zwar zum beiderseitigen Vorteil der Erzeuger und der Nutzer technischen Wissens, auf eine dem gesellschaftlichen und wirtschaftlichen Wohl zuträgliche Weise sowie zu einem Gleichgewicht der Rechte und Pflichten.

Laut Artikel 27 des Abkommens werden Patente für alle Erfindungen, ob es sich um Erzeugnisse oder Verfahren handelt, auf allen Gebieten der Technik erteilt, sofern sie neu sind, auf einer erfinderischen Tätigkeit beruhen und gewerblich anwendbar sind. Die Erteilung von Patenten und die Ausübung von Patentrechten erfolgt ohne Diskriminierung aufgrund des Ortes der Erfindung, des Gebiets der Technik oder danach, ob die Erzeugnisse eingeführt oder an Ort und Stelle hergestellt werden.

Von der Patentfähigkeit ausgeschlossen werden können jedoch Erfindungen, die als Gefahr für die öffentliche Ordnung und die guten Sitten gelten, sowie diagnostische, therapeutische und chirurgische Verfahren für die Behandlung von Menschen und Tieren. Pflanzensorten und Tierarten mit Ausnahme von Mikroorganismen und im wesentlichen biologische Verfahren zur Züchtung von Pflanzen und Tieren mit Ausnahme von nichtbiologischen und mikrobiologischen Verfahren können ebenfalls ausgenommen werden. Für den Fall der Pflanzensorten haben die Mitglieder einen Schutz «entweder durch Patente oder durch ein wirksames System eigener Art oder durch eine Verbindung beider» vorzusehen.

In Artikel 28 werden die ausschliesslichen Rechte des Patentinhabers beschrieben. Danach ist es Dritten untersagt, ohne Zustimmung des Inhabers die patentierte Erfindung, ob ein Erzeugnis oder ein Verfahren, herzustellen, zu verwenden oder zu verkaufen. Patentinhaber sind auch berechtigt, das Patent rechtsgeschäftlich oder im Wege der Rechtsnachfolge zu übertragen und Lizenzverträge zu schliessen.

Die Artikel 65 und 66 behandeln die besonderen Vereinbarungen für die Entwicklungsländer als Mitglieder, vor allem die Übergangsvereinbarungen und die «Gnadenfrist», welche diese Länder zur Erfüllung ihrer Verpflichtungen erhalten. Die Industrieländer unter den Mitgliedern müssen die Bestimmungen des Abkommens in einer Frist von einem Jahr nach dem Inkrafttreten (1. Januar 1995) des WTO-Abkommens anwenden. Die Entwicklungsländer sind berechtigt, diese Frist um weitere vier Jahre zu verschieben. 
Für die am wenigsten entwickelten Mitglieder wird eine noch längere Frist von 10 Jahren eingeräumt, bevor sie die TRIPS-Bestimmungen anwenden müssen. Der Rat für TRIPS kann sogar eine zusätzliche Verlängerung bewilligen.

Des weiteren ermöglicht eine TRIPS-Bestimmung die Erteilung von Zwangslizenzen (d.h. eine Lizenz wird ohne die Zustimmung des Patentinhabers vergeben, siehe oben Abschnitt 2). Zwangslizenzen können einem Anmelder zur Verwendung einer patentierten Erfindung erteilt werden, wenn der Patentinhaber eine freiwillige Lizenz zu angemessenen kommerziellen Bedingungen innerhalb einer vernünftigen Frist verweigert. Allerdings werden zum Schutz der berechtigten Interessen des Patentinhabers verschiedene Bedingungen vorgeschrieben. In Fällen der nichtgewerblichen Benutzung im öffentlichen Interesse oder im Fall des nationalen Notstands kann ausnahmsweise eine Befreiung von der Pflicht, zuerst eine freiwillige Lizenz zu beantragen, gewährt werden.

\subsection{BESONDERE TRIPS-BESTIMMUNGEN ÜBER DEN TECHNOLOGIETRANSFER}

Neben dem allgemeinen oben erwähnten Ziel, Innovation, Transfer und Verbreitung neuer Technologien zu fördern, fordert das TRIPS-Abkommen die Industrieländer gezielt auf, für ihre Unternehmen und Institutionen Anreize zu schaffen, um den Transfer von Technologie in die am wenigsten entwickelten Mitglieder zu unterstützen (Artikel 66).

Die Bedingung der Offenbarung für einen Patentantrag (vom Anmelder eines Patents wird verlangt, die Erfindung so deutlich und vollständig zu offenbaren, dass ein Fachmann sie ausführen kann - Artikel 29) hat eine Reihe wichtiger Auswirkungen. Erstens werden so Informationen über die Herkunft der Technologie während der Schutzdauer leicht erhältlich. Zweitens geht ein Patent nach Ablauf der Schutzfrist in Gemeingut über und wird allgemein frei verfügbar. Artikel 30 schliesslich bezieht sich auf «begrenzte Ausnahmen» von den Rechten aus dem Patent und ermöglicht so die Verwendung einer Erfindung zu Versuchs- oder, anders ausgedrückt, Forschungszwecken.

Wenn eine Regierung über (patentrechtlich geschützte oder ungeschützte) Technologien verfügt, so darf sie diese laut TRIPS-Abkommen zu konzessionellen Bedingungen übertragen, sofern sie dies wünscht. Analog dazu verbietet das TRIPS-Abkommen Regierungen oder internationalen Finanzinstitutionen nicht, finanzielle Unterstützung zu leisten, um den freiwilligen Transfer von rechtlich geschützter Technologie eines privaten Eigentümers zu konzessionellen Bedingungen zu ermöglichen.

\subsection{OPTIONEN DER ENTWICKLUNGSLÄNDER FÜR DEN SCHUTZ DES GEISTIGEN EIGENTUMS}

Die meisten Entwicklungsländer sehen zum Schutz des gewerblichen Eigentums Patente vor. Bislang haben freilich erst wenige einen Schutz des geistigen Eigentums für genetisches Material von Pflanzen und/oder für Pflanzensorten eingeführt. Diesen Ländern bietet die Verpflichtung aus dem TRIPS-Abkommen verschiedene Möglichkeiten:

๑ Patentschutz für das gesamte genetische Material, einschliesslich Pflanzensorten; 
$\square$ zwei Schutzsysteme (wie in den Vereinigten Staaten): Patentschutz für genetisches Material von Pflanzen und das Züchterrecht oder ein System eigener Art (sui generis) zum Schutz von Pflanzensorten;

- Ausschliessung des pflanzlichen Genmaterials von der Patentfähigkeit und Erarbeiten eines sui generis-Systems oder Beitritt zur UPOV.

\section{GEISTIGE EIGENTUMSRECHTE, BIOTECHNOLOGIE UND LANDWIRTSCHAFT}

Am Beispiel der Biotechnologie in der Landwirtschaft prüft dieser Abschnitt die Frage, ob die Stärkung und Ausweitung der geistigen Eigentumsrechte den Technologietransfer und die Innovation in der Landwirtschaft der Entwicklungsländer erleichtern oder erschweren wird.

Zunächst müssen zwei Typen von Technologietransfer in der Biotechnologie auseinandergehalten werden. Im ersten Fall handelt es sich um einen Transfer (Einfuhr, direkter Kauf) des Enderzeugnisses der Biotechnologie (z.B. neue genmodifizierte Sorte). Im zweiten Fall wird die Biotechnologie als Instrument in der Agrarforschung eingesetzt (z.B. genetische Marker in der Pflanzenzüchtung). Die Auswirkungen der geistigen Eigentumsrechte auf den Technologietransfer sind je nach Fall - biotechnologische Produkte oder F \& E - in Art und Ausmass sehr unterschiedlich.

\subsection{AUSWIRKUNGEN AUF LANDWIRTE UND SONSTIGE ENDVERBRAUCHER}

Das schlagkräftigste Argument für eine Stärkung der geistigen Eigentumsrechte ist die Förderung von Investitionen und Innovation. Stimmt das, so dürfte der Technologietransfer an Landwirte oder sonstige Endverbraucher diesen eine breitere Palette neuer Technologien zur Verfügung stellen. Grundsätzlich sollte dies für die landwirtschaftlichen Erzeuger positive Folgen haben. Gleichzeitig könnten Unternehmen davon absehen, rechtlich geschützte Technologie an Länder zu liefern, in denen ein wirksames Schutzsystem für das geistige Eigentum fehlt.

Eindeutige Tendenzen zu den Preisen der neuen genmodifizierten Sorten lassen sich noch nicht erkennen, da die Erfahrungen sich erst auf wenige Länder beschränken (darunter Australien, Kanada, Frankreich, Vereinigte Staaten) und zudem sehr jungen Datums sind (Brenner, 1997). In manchen Fällen kostet genmodifiziertes Saatgut nicht mehr als konventionelles; in anderen wiederum beinhaltet der Kauf von Saatgut eine «Technologieprämie». Allgemein ist anzunehmen, dass die Kosten der neuen genmodifizierten Sorten jene der nach traditionellen Methoden hergestellten Sorten übersteigen werden. Landwirte, welche die neuen genmodifizierten Sorten erwerben, müssen die Preiserhöhung gegen die Vorteile der neuen Sorte - Qualität, Ertrag oder Seuchen- und Krankheitsresistenz - abwägen. Ob das Saatgut als geistiges Eigentum geschützt ist oder nicht ist für die Landwirte nicht relevant, ausser wenn dadurch ihre vorherigen Rechte auf Aufbewahrung, Wiederverwendung oder Austausch von Erntegut beschnitten werden.

Die Erfahrungen in Indien mit Hybrid-Maissaatgut (Hybrid-Sorten verleihen den Pflanzenzüchtern einen patentähnlichen Schutz) sind sehr aussagekräftig. Obwohl 
das privatwirtschaftlich produzierte Hybrid-Saatgut wesentlich teurer war als die vom öffentlichen Sektor erzeugten, verbesserten offen bestäubten Sorten (OPV), stieg der private Verkauf von Hybrid-Saatgut deutlich rascher an als der Absatz von OPV. Der Anteil des privatwirtschaftlichen Verkaufs von Maissaatgut liegt heute über 50 Prozent. Das führt zum Schluss, dass Privatunternehmen bei der Lieferung des Materials an die Landwirte entweder effizienter arbeiten und/oder dass sie Saatgut von beständigerer Qualität anbieten (Singh et. al. 1995).

\subsection{AUSWIRKUNGEN FÜR LOKALE INNOVATOREN}

Ein Schlüsselthema für Entwicklungsländer ist die Frage, ob der Schutz geistigen Eigentums die Rolle der lokalen Innovatoren fördern und damit die nationale Innovationskapazität stärken wird.

Sowohl in den OECD-Staaten als auch in Entwicklungsländern verändern sich die Rollen und das Gleichgewicht von öffentlichem und privatem Sektor im landwirtschaftlichen Innovationsprozess. In den OECD-Ländern haben in den letzten Jahren öffentliche Beiträge zur Finanzierung der Agrarforschung und Innovation stagniert oder abgenommen. Von 1981 bis 1983 zum Beispiel legte die private Agrarforschung jährlich 5,1 Prozent zu und machte am Ende dieses Zeitraums rund die Hälfte aller Agrarforschungsausgaben aus (Pardey, 1997). Eine besonders wichtige Rolle spielten die privatwirtschaftlichen Investitionen in die Biotechnologie.

Aufgrund des vorhandenen Erfahrungsmaterials ist anzunehmen, dass geistige Eigentumsrechte einen Anreiz für Investitionen des Privatsektors in die Agrarforschung darstellen (Thirtle and Echeverria, 1994; Fuglie et. al. 1996). Allerdings ist auch zu erkennen, dass private Investitionen in die Pflanzenzüchtung sich wahrscheinlich auf eine begrenzte Anzahl kommerzieller Fruchtarten wie Baumwolle, Mais, Sojabohnen, Gemüse und Weizen beschränken werden. Privatwirtschaftliche Investitionen in F \& E für OPV und für die Kulturen von Landwirten mit wenig Ressourcen gelten als unwahrscheinlich, es sei denn, dass dafür Anreize geschaffen werden.

\subsection{AUSWIRKUNGEN AUF DIE ÖFFENTLICHE FORSCHUNG}

Der Schutz geistiger Eigentumsrechte wird erwartungsgemäss positive wie negative Folgen für die öffentlichen Agrarforschungssysteme zeitigen. In zahlreichen Entwicklungsländern spielt der öffentliche Sektor in der Agrarforschung im allgemeinen und in der biotechnologischen Forschung im besonderen, aber auch bei der Technologieentwicklung und im Vertrieb weiterhin die Schlüsselrolle. Als Folge der Strukturanpassung und der Liberalisierung, die in immer mehr Ländern durchgeführt werden, haben jedoch öffentliche Investitionen in die Agrarforschung stagniert oder gar abgenommen. So stehen öffentliche Forschungseinrichtungen zunehmend unter Druck, sich auf einkommenerzeugende Aktivitäten umzuorientieren. Die Einführung des Schutzes geistiger Eigentumsrechte könnte sich insofern positiv auswirken, als Einrichtungen, die ihre «Innovationen » bislang dem staatlichen und privaten Sektor frei zur Verfügung stellten, dank den Lizenzen, Gebühren oder dem Dienstleistungsangebot nun neu Einkommen erzielen (van Wijk and Jaffé, 1995). 
Andererseits leisteten bisher die Ausbildung in Forschungseinrichtungen und Universitäten der Industrieländer sowie die Teamforschung einen wesentlichen Beitrag zur Agrarforschung der Entwicklungsländer. Die Wissenschaftler der OECD-Länder äussern heute die Befürchtung, dass der zuvor in der akademischen Forschung übliche freie Austausch von wissenschaftlichen Informationen und Erkenntnissen allmählich verschwinden wird. Dafür sind zwei Faktoren verantwortlich: Erstens sind öffentliche Forschungseinrichtungen immer stärker auf private Finanzierung angewiesen; zweitens entwickelt sich ein immer grösserer Teil der wissenschaftlichen Ergebnisse zu Kenntnissen, für welche die Universität selbst oder mit einem oder mehreren privaten Partnern geistige Eigentumsrechte beansprucht. Dies hat selbstverständlich Folgen für die Wissenschaftler in Entwicklungsländern.

In der Diskussion um die geistigen Eigentumsrechte wurde die Besorgnis laut, die Ausdehnung des Schutzes geistiger Eigentumsrechte auf genetisches Material könne den Austausch von Keimzellenplasma - ein wichtiger Input für F \& E in der Landwirtschaft - behindern. Befürworter der geistigen Eigentumsrechte argumentieren, der Schutz fördere den Transfer von Genmaterial aus Industriein Entwicklungsländer, was allerdings zu überprüfen bleibt. Angesichts des starken privatwirtschaftlichen Engagements in der Biotechnologie ist abzusehen, dass ein steigender Anteil der neuen Biotechnologien unter rechtlichen Schutz (eine Art der geistigen Eigentumsrechte) gestellt wird. In diesem Fall führt der Schutz des geistigen Eigentums möglicherweise zu einem Anstieg des Genmaterial-Transfers in einem marktgeprägten kommerziellen Umfeld. Ungeklärt bleibt indessen, wie es sich mit dem Material verhält, das in einem nicht-marktgeprägten Umfeld (öffentliches Gut) gehandelt wird.

Rechtlich geschützte Technologie kann über verschiedene Wege aus kommerziellen und nicht kommerziellen Quellen bezogen werden. Neben dem direkten Verkauf oder der Lizenz bildet auch die Prüfung der Patentanträge wegen des Erfordernisses der Offenbarung eine wertvolle Quelle für technologische Informationen. Das Patentrecht und das Sortenschutzrecht sehen auch eine Ausnahme für die Forschung vor: Das Ausgangsmaterial einer rechtlich geschützten privaten Erfindung kann so zu Forschungszwecken verfügbar gemacht werden.

Gemeinsame öffentliche Programme und Projekte, z.B. zwischen Universitäten, erleichtern den Zugang zu rechtlich geschützter Technologie, ebenso bilaterale und multilaterale Entwicklungshilfe oder die Internationalen Agrarforschungszentren (IARC). Gleiches gilt für Stellen wie den International Service for the Acquisition of Agri-Biotech Applications (ISAA), die beim Transfer oder der unentgeltlichen Überlassung von rechtlich geschützter Technologie an den öffentlichen Sektor als Vermittler oder «Technologie-Makler» wirken.

Beispiele solcher Schenkungen sind erstens das Abkommen zwischen ISAA in Monsanto und dem «Centre of Research and Advances Studies» (CINEVESTAV) in Mexiko, zweitens das Abkommen zwischen Ciba-Geigy (heute Novartis) und dem «International Rice Research Institute» (IRRI), ein IARC, in den Philippinen. Gegenstand des ersten Abkommens ist der Transfer patentierter Gentechnologie zur Entwicklung von Virusresistenz in Kartoffeln von Monsanto an eine staatliche mexikanische Forschungseinrichtung. Im zweiten Abkommen geht es um ein von Ciba-Geigy patentiertes synthetisches Bacillus thuringiensis (Bt.)-Gen zur Entwicklung von Insektenresistenz, das dem IRRI für die Verwen- 
dung bei genmodifiziertem Reis überlassen wird. In beiden Beispielen wurde der Transfer im Rahmen einer gebührenfreien Lizenz abgewickelt. Im zweiten Fall darf der mit dem Bt.-Gen erzeugte Reis nicht Produzenten in Australien, Kanada, Japan, Neuseeland, den Vereinigten Staaten und an Unterzeichner des Europäischen Patentübereinkommens angeboten werden.

Heute sind nur wenige Beispiele von Technologie-Schenkungen (mit oder ohne Dienste eines Vermittlers) bekannt. Erfolg oder Scheitern solcher Initiativen kann noch nicht definitiv beurteilt werden.

Offensichtlich sind jedoch gewisse Änderungen beim Austausch von Keimzellenplasma: die rechtlichen und/oder kommerziellen Vereinbarungen machen aus dem zuvor freien Verkehr einen Transfer. Unklar bleibt, wie die neuen Vereinbarungen zum Technologietransfer das Austauschvolumen beeinflussen werden.

Unter dem Strich dürfte die Einführung oder Stärkung der geistigen Eigentumsrechte in Biotechnologie und Landwirtschaft für Landwirte, lokale Innovatoren oder für die nationale Agrarforschung positive und negative Folgen haben. Eine wichtige ungelöste Frage betrifft die Rolle der Internationalen Agrarforschungszentren (IARC) der Konsultativen Gruppe für internationale landwirtschaftliche Forschung (CGIR, allgemein als CG-System bezeichnet). Die IARC haben zum Transfer von Technologien der Grünen Revolution an Entwicklungsländer - vor allem ertragreiche Weizen- und Reissorten - entscheidend beigetragen.

Die IARC, die im Oktober 1994 ein Abkommen mit der FAO unterzeichneten, verwalten in ihren Sammlungen die pflanzlichen genetischen Ressourcen als «Verwahrer» treuhänderisch für die internationale Gemeinschaft. Die Zentren stellten früher Keimzellenplasma aus Genbanken und Material aus eigener Forschung den Entwicklungs- und Industrieländern, Forschungsinstitutionen des öffentlichen und privaten Sektors und Unternehmen unentgeltlich zur Verfügung. Mit der Erweiterung der geistigen Eigentumsrechte gerät das CG-System in eine heikle Lage: Dritte können für Sorten, die aus dem von den Zentren gelieferten Material abgeleitet werden, Patentschutz oder Sortenschutz erhalten. Gleichzeitig stehen die Zentren vor dem Dilemma, gegebenenfalls durch private Unternehmen patentiertes fortgeschrittenes Material oder Forschungstechniken als wichtigen Beitrag an ihre eigenen Forschungsarbeiten entgegenzunehmen.

Die Stärkung der geistigen Eigentumsrechte wirft folgende Frage auf: Inwiefern wird ein gutes öffentliches System für Forschung und Technologietransfer zugunsten der Entwicklungsländer (wie bei der Grünen Revolution) neben einem zunehmend globalen System rechtlich geschützter Technologie bestehen können?

\section{SCHLUSSFOLGERUNGEN}

In den vorhergehenden Abschnitten wurden am Beispiel der Biotechnologie in der Landwirtschaft mögliche Auswirkungen der geistigen Eigentumsrechte auf die Entwicklungsländer untersucht. Dabei ist zu betonen, dass die Erforschung der Konsequenzen der geistigen Eigentumsrechte auf Technologietransfer und Innovation in Entwicklungsländern erst in den Anfängen steckt. Ferner muss klar sein, dass geistige Eigentumsrechte an sich nicht ausreichen, um den Technologietransfer und die Innovationen anzukurbeln, wenn weitere unterstützende Massnahmen und Institutionen fehlen. 
Einige Forscher äusserten denn auch Zweifel an den Vorteilen einer Einführung geistiger Eigentumsrechte für die Entwicklungsländer. Um z.B. die Beziehung zwischen globaler Patentherstellung und Wohlstand zu untersuchen, analysiert ein Autor an einem einfachen Patentmodell die Wohlstandseffekte der Ausdehnung des Patentschutzes vom «Erfinderland» auf das «Verbraucherland». Die Studie kommt zum Schluss, dass mit der immer breiteren Ausdehnung des Patentschutzes auf immer mehr Länder die Wohlstandseffekte insgesamt abnehmen und sogar negative Werte erreichen. Daraus folgt, dass zumindest die Länder mit den niedrigsten Einkommen von neuen Vereinbarungen über die Erweiterung des Patentschutzes ausgenommen werden sollten (Deardoff, 1992).

Eine umfassende Untersuchung der Fachliteratur (Seibeck et. al. 1990) stellt fest, dass geistige Eigentumsrechte für Entwicklungsländer je nach Land und wahrscheinlich je nach Sektor unterschiedliche Vor- und Nachteile bringen werden. Beeinflusst wird das Kosten-Nutzen-Verhältnis voraussichtlich durch folgende Faktoren: Reaktion der inländischen Innovatoren auf den höheren Schutz; Reaktion der ausländischen Direktinvestoren; Nachfrage für geschützte Produkte.

Trotz der Tendenz zur Stärkung und Erweiterung der geistigen Eigentumsrechte bleiben weite Bereiche der vorhandenen Technologie Gemeingut - entweder, weil nie ein Schutztitel beantragt wurde, oder weil die Schutzfrist abgelaufen ist. Allerdings gilt als sicher, dass in der Ära nach dem TRIPS die Technologie vermehrt geschützt und der Transfer zunehmend zu Marktbedingungen erfolgen werden. Es fragt sich, wie die Chancen für eine «gute öffentliche» Technologie stehen, für welche in den Entwicklungsländern zwar ein grosser Bedarf (öffentliches Gesundheitswesen, Umweltschutz, Ernährung, Landwirtschaft), aber zumindest kurzfristig keine Nachfrage im ökonomischen Sinn besteht.

Organisationen, die Projekte und Programme zur Entwicklung oder für den Transfer "guter öffentlicher» Technologie zugunsten der Entwicklungsländer durchführen (bilaterale und multilaterale Organisationen für Entwicklungshilfe, private Stiftungen, NRO) sollten daher unbedingt wissen, welche Probleme als Folge der breiten Anwendung geistiger Eigentumsrechte zu erwarten sind. Mit diesem Bewusstsein gewappnet werden sie mögliche Hindernisse und Sachzwänge besser vorhersehen und sich Strategien zurechtlegen können, um die Schwierigkeiten zu überwinden.

Auch die Regierungen der Entwicklungsländer spielen bei der Schaffung eines angemessenen Regelumfelds für geistige Eigentumsrechte und bei der Definition einer geeigneten Politik zur Förderung von Technologietransfer und Innovationen eine Schlüsselrolle. Solche politischen Entscheidungen sind sehr komplex. Berücksichtigt werden muss dabei :

\subsection{VERPFLICHTUNGEN AUS DEM TRIPS-ABKOMMEN EINHALTEN}

Artikel 27 (3b) des TRIPS-Abkommens, welcher den Schutz von Pflanzensorten vorsieht, soll im Januar 1999 revidiert werden. Die Entwicklungsländer unter den Unterzeichnerstaaten haben also nur sehr wenig Zeit, um sich für eine der möglichen Schutzformen zu entscheiden und um auf ihre besonderen Bedürfnisse und Interessen abgestimmte Schutzsysteme zu erarbeiten. Unter der Ägide des IPGRI wurden erste Bemühungen unternommen, um mögliche Inhalte einer 
mit dem TRIPS kompatiblen sui generis-Alternative zur Mitgliedschaft in der UPOV zu bestimmen und zu evaluieren (Leskien and Flintner, 1997).

In manchen Ländern dürfte das Recht des geistigen Eigentums nur geringfügige Änderungen existierender Gesetze erfordern, in anderen jedoch müssen möglicherweise neue Institutionen und Strukturen geschaffen werden. Eine Gesetzgebung zum geistigen Eigentum bedingt auch die Implementierung, Überwachung und Durchsetzung. Dementsprechend sind finanzielle Mittel, technisches und juristisches Fachwissen erforderlich.

\subsection{GEEIGNETE RAHMENBEDINGUNGEN FÜR LOKALE INVESTOREN SCHAFFEN}

Ein wirksamer Schutz des geistigen Eigentums dürfte einen Anreiz für Forschung und Innovation des Privatsektors bilden. Dazu müssen jedoch die einschlägigen Gesetze tatsächlich angewandt werden, wofür in der Regel nationale Institutionen über die Patentämter oder, für den Falls des Sortenschutzes, Organisationen des Saatgutsektors zuständig sind. In zahlreichen Ländern müssen diese Einrichtungen besser entwickelt werden. Neben der Gesetzgebung zum geistigen Eigentum ist für die Biotechnologie ein klares Regelungsumfeld für den sicheren Transfer neuer biotechnologischer Produkte notwendig. Im übrigen haben bislang erst wenige Entwicklungsländer Leitlinien oder Gesetze zur biologischen Sicherheit verabschiedet.

In Ländern mit schwach entwickelten Technologiemärkten sind zusätzliche Anreize nötig, damit der Privatsektor sich engagiert. Ferner sollte man sich verstärkt bemühen, den Privatsektor durch die Schaffung von öffentlich/privaten «Partnerships» einzubeziehen.

\subsection{ROLLE DES ÖFFENTLICHEN SEKTORS BESTIMMEN}

Um die nationale Innovation zu fördern, muss die öffentliche Forschung künftig gezielter eingesetzt werden. Da kurzfristig eine Zunahme der öffentlichen Investitionen in Forschung (national und durch Geldgeber finanziert) als unwahrscheinlich gilt, ist es entscheidend wichtig, die knappen öffentlichen Mittel effizient einzusetzen. In einigen Ländern übernimmt die Privatwirtschaft bereits eine aktivere Rolle im Bereich Forschung und Entwicklung. Diese Tendenz sollte gefördert werden.

Selbst nach der Einführung eines Systems geistiger Eigentumsrechte werden bestimmte Forschungsgebiete und Wirtschaftssektoren kurzfristig kaum Privatinvestitionen anziehen. Deshalb sollte der öffentliche Sektor in Sachen Forschung und Innovation zwei Aufgaben erfüllen: Mitarbeit durch eine Förderung der privatwirtschaftlichen Beteiligung (wo möglich); Konzentration der Forschung auf sozial und ökologisch vorrangige Gebiete, die geringe Aussichten auf kurzfristige private Gewinne bieten.

\subsection{TECHNOLOGIETRANSFER-ABKOMMEN AUSHANDELN}

Selbst bei geschützter Technologie besteht für Regierungen oder staatliche Stellen ein gewisser Verhandlungsspielraum. Keine Bestimmung des TRIPS- 
Abkommens verbietet es den Regierungen (einem Geber, einer internationalen Finanzinstitution), günstige Bedingungen auszuhandeln und/oder finanzielle und technische Hilfe für den nicht kommerziellen Transfer von rechtlich geschützter Technologie zu vereinbaren.

Daneben haben die Regierungen auch die Möglichkeit, sich an einen «ehrlichen Makler» zu wenden - Organisationen wie die ISAAA, NRO oder unabhängige Sachverständige - der in den Verhandlungen zu den Bedingungen des Technologietransfers zwischen Regierungen und öffentlichen Behörden und den Privatunternehmen vermittelt.

\section{BIBLIOGRAPHIE}

Brenner, Carliene (1996), Integrating Biotechnology in Agriculture: Incentives, Constraints and Country Experiences, Development Centre Studies, OECD, Paris.

Brenner, Carliene (1997), Biotechnology Policy for Developing Country Agriculture, Policy Brief No. 14, OECD Development Centre, Paris.

Brenner, Carliene, Intellectual Property Rights and Technology Transfer in Developing Country Agriculture: Rhetoric and Reality, OECD Development Centre, Paris, forthcoming.

Deardorff, Alan V. (1992), «Welfare Effects of Global Patent Protection », in Economica, Vol. 59, February

Echeverria, Rubin, Eduardo J. Trigo and Derek Byerlee (1996), Institutional Change and Effective Financing of Agricultural Research in Latin America, World Bank Technical Paper No. 330, Washington, D.C. August.

Fuglie, Keith, Nicola Ballenger, Kelly Day, Cassandra Klotz, Michael Ollinger, John Reilly, Uptal Vasavada and Jet Yee (1996), Agrigultural Research and Development: Public and Private Investments under Alternative Markets and Institutions, United Sttes Department of Agriculture, AER No. 735, Washington, May.

Joly, Pierre-Benoît and Marie-Angèle de Looze (1996), «An Analysis of Innovation Strategies and Industrial Differentiation through Patent Applications: the Case of Plant Biotechnology ", in Research Policy, Vol. 25.

Juma, Calestous (1989), The Gene Hunters: Biotechnology and the Scramble for Seed, Princeton University Press, Princeton.

Leskien, Dan and Michael Flitner (1997), Intellectual Property Rights and Plant Genetic Ressources: Options for a Sui Generis System, IPGRI, Issues in Genetic Ressources No. 7, Rome.

Lesser, W. (1991), Equitable Patent Protection in the Developing World: Issues and Approaches, Eubios Ethics Institute, New Zealand.

Lesser, W. (1994), Institutional Mechanisms supporting Trade in Genetic Materials, UNEP, Environment and Trade Series No. 4, Geneva.

Lesser, W. (1997), Assessing the Implications of IPR on Plant and Animal Agriculture, Staff Paper 97-04, Cornell University, Ithica, U.S.A., June.

OECD (1996), Intellectual Property, Technology Transfer and Genetic Resources, Paris.

Pardey, Phillip (1997), IFPRI, Principles, Policies and Institutional Aspects of Financing Agricultural R \& D, paper presented at the workshop on «New Investment Strategies for Agricultural and Natural Resources Research », London, 1 - 2 October, 1997

Siebeck, Wolfgang E. with Robert Evenson, William Lesser and Carlos A. Primo Braga (1990), Strenghtening Protection of Intellectual Property in Developing Countries : A Survey of the Literature, World Bank Discussion Paper No. 112.

Singh, R.P., Suresh Pal and Michael Morris (1995), Maize Research, Development and Seed Production in India: Contributions of the Public and Private Sector, Economics Working Paper 9503, CIMMYT, Mexico.

Thirtle, Colin and Ruben G. Echeverria (1994), Privatisation and the Roles of Public and Private Institutions in Agricultural Research in Sub-Saharan Africa, in: Food Policy, Vol. 19, No. 1, February.

van Wijk, Jeroen, and Walter Jaffé (eds.) Proceedings of a Seminar on the Impact of Plant Breeders'Rights in Developing Countries, March 7-8, 1995, Bogota, Colombia. 